\title{
Analysis of Bus Power Balance Based on Mathematical Statistics
}

\author{
Fujun Bao", Miao Wang, Qiang Li, Lei Wang and Yansheng Lang \\ China Electric Power Research Institute, China \\ *Corresponding author
}

\begin{abstract}
The bus power balance is an important index to quantify the power system basic data. In view of the current shortage of power imbalance evaluation at single time section, in this paper, imbalanced power is regarded as a normal distributed random variable and the its historical data is statistically analyzed. This method takes the sample median as the estimate of the population mean, confidence interval of sample standard deviation as the interval estimation of population standard deviation, reflecting the distribution of bus imbalanced power. An example on actual stations is given in this paper, the power system basic data are quantified from the perspective of statistics.
\end{abstract}

Keywords-power balance; normal distribution; basic data; historical data; sample median; confidence interval

\section{INTRODUCTION}

Basic data quality of power system is the key factor which affects the application of the smart grid dispatch and control system. There are many factors that affect the quality of measurement data, such as accuracy degree of electric transformer, size of telemetry dead zone, communication delay and scanning period of full data. So we can find the existing problems of basic data from the data evaluation. The current researches are concentrate on the measurement accuracy improvement, but less on the research of data evaluation.

At present, the data quality is evaluated from these two aspects: the measurement data and state estimation results. Because the true value of equipment measurement is not available, the evaluation of the state estimation results is generally based on the difference between the measured value and the estimated value. Despite the true value is unknown, document [6] put forward the evaluation index of state estimation results based on measurement uncertainly theory. In view of the deficiency of single evaluation index, document [7] puts forward a multi index comprehensive evaluation method, which reflects the problems of power grid from various angles. According to the evaluation of the state estimation results, it can reflect the model, topology, equipment parameters, measurement and other aspects of the power grid. But due to the influence of the model and algorithm, the evaluation cannot fully reflect all aspects of basic data quality, so we need to find other methods to supplement the evaluation of state estimation results.

In 2015, balanced qualified rate of bus power was added as an evaluation index of the dispatching automation system. Select the SCADA measurement data as the data source, balanced qualified rate of bus power is designed to improve the quality of basic data. This index has the following characteristics. It is not affected by the model, parameters or other factors. Due to the data acquisition from the same station, the influence of transmission delay is small.

State Grid Corporation of China dispatching control center publish the Practical requirements of smart grid dispatching control system and Practical acceptance method of smart grid dispatching control system. These two regulations add the node power balanced rate as an evaluation index of power grid operation, considering the bus power balance and the active power balance of branch. Based on a time section of bus imbalanced power, the conventional evaluation method can give good or bad results in a qualified rate threshold. But the conventional evaluation results do not have discrimination. In fact, due to the influence of measuring device, network equipment and other factors, the qualified rate of a uniform threshold does not fully reflect the quality of basic data.

Take the historical data of the bus unbalanced power as sample, this paper can give the fluctuation of each bus imbalanced power based on the mathematical analysis of historical data. In this paper, the quality of the overall data is reflected by the evaluation of those samples in a period of time, so that it can better grasp the change of measurement. At the end of this paper, the practical application of index of bus power balance is introduced.

\section{NORMAL DISTRIBUTED RANDOM VARIABLE}

In general, in the $500 \mathrm{kV}$ or $220 \mathrm{kV}$ substation, the power flow into the bus should be balanced with the power output. The algebraic sum of active power/reactive power on the bus bar should be close to zero. In the automation system, the value of active power and reactive power which is monitored by the dispatching station is from the measured value of each line measurement and control device in the remote substation. Because there is measurement error in the measurement and control device, the algebraic sum of active power/reactive power on the bus bar are fluctuating, which are monitored by the dispatching master station.

For a single device, the deviation between measured and true values:

$$
X_{i}=Z_{i}-Z_{T i}
$$


$X_{i}$ is a normal distributed random variable with a mean value of $\mu_{i}=0$ and a standard deviation of $\sigma_{i}$.

$$
X_{i} \sim N\left(0, \sigma_{i}^{2}\right) \quad i=1,2, \ldots, n
$$

$Z_{i}$ is the observed value of device measurement, $Z_{T i}$ is the true value of device measurement, $i$ is the device serial number, $n$ is the number of device, which measure the power inflow and outflow of the bus.

Because the true value of device measurement cannot be acquired, so the evaluation of a single device measurement is not accurate. The algebraic sum of all the true value of the device measurement connected to the bus bar is:

$$
\sum_{i=1}^{n} Z_{T i}=0
$$

The bus imbalanced power is $Y_{j}$.

$$
Y_{j}=\sum_{i=1}^{n} Z_{i}=\sum_{i=1}^{n}\left(X_{i}+Z_{T i}\right)=\sum_{i=1}^{n} X_{i} \quad j=1,2, \ldots, m
$$

In the formula, $m$ is the amount of bus, $j$ is the serial number of bus.

According to the characteristics of the normal distributed random variables, the superposition of the normal distribution is still normal distribution. So $Y_{j}$ is a normal distributed random variable with a mean value of $\mu_{j}=0$ and a standard deviation of $\sigma_{j}$.

$$
Y_{j} \sim N\left(0, \sum_{i=1}^{n} \sigma_{i}^{2}\right) \sim N\left(0, \sigma_{j}^{2}\right)
$$

$Y_{j}$ is statistics, and it can be observed. Each measurement is independent. In the statistical results of historical data, we make the evaluation of the bus power balance according to the size of sample mean $\mu_{j}$ and standard deviation $\sigma_{j}$. Compared with the evaluation by single calculation result, this method is more reasonable.

\section{MATHEMATICAL ANALYSIS}

\section{A. Interval Estimation}

In the distribution of $Y_{j}$, the standard deviation $\sigma_{j}$ is unknown. But through the estimation, we can get the estimated value. Because it does not know the true value of the unknown parameter $\sigma_{j}$, it is difficult to determine the size of the error. Interval estimation is given a confidence interval that can contain the true value of unknown parameters in the given confidence level.

If statistics $\theta_{1}$ and $\theta_{2}$ meet the following conditions:

$$
P_{\theta}\left\{\theta_{1} \leq \theta \leq \theta_{2}\right\} \geq 1-\alpha
$$

When the confidence level is $1-\alpha, \theta_{1}$ is the confidence lower limit of the parameter $\theta$, and $\theta_{2}$ is the confidence upper limit of the parameter $\theta$. This indicates that the probability of interval $\left[\theta_{1}, \theta_{l}\right]$ contains the parameter $\theta$ true value is not less than 1- $\alpha$. Therefore, in the same confidence level, the lower the interval ranges, the higher the accuracy of the interval estimation.

While population mean value $\mu_{j}$ of bus imbalanced power is 0 , the confidence lower limit $\theta_{1}\left(\sigma_{j}^{2}\right)$ and the confidence upper limit $\theta_{2}\left(\sigma_{j}^{2}\right)$ of population mean square deviation $\sigma_{j}^{2}$ is :

$$
\theta_{1}\left(\sigma_{j}^{2}\right)=\frac{\sum_{i=1}^{N}\left(Y_{j}^{(i)}-\mu_{j}\right)^{2}}{\chi^{2}\left(N, \frac{\alpha}{2}\right)} \quad \theta_{2}\left(\sigma_{j}^{2}\right)=\frac{\sum_{i=1}^{N}\left(Y_{j}^{(i)}-\mu_{j}\right)^{2}}{\chi^{2}\left(N, 1-\frac{\alpha}{2}\right)}
$$

In this formula, $j$ is the serial number of bus, $N$ is the amount of sample, $i$ is the serial number of sample, $\chi^{2}(N)$ is the chi square distribution with freedom degree $N$. So the interval estimation of mean square deviation $\sigma_{j}^{2}$ is $\left[\theta_{l}\left(\sigma_{j}^{2}\right), \theta_{2}\left(\sigma_{j}^{2}\right)\right]$.

\section{B. Sample Median}

Bus imbalanced power $Y_{j}$ is a normal distributed random variable with a mean value of $\mu_{j}=0$ and a standard deviation of $\sigma_{j}$. Due to the measurement uncertainty, the measurement error is unavoidable, and the samples mean value $\mu_{j}$ may not be 0 . The difference between the sample mean value $\mu_{j}$ and 0 represents the deviation between the bus imbalanced power and the standard mean. In order to reduce the influence of the individual abnormal data on the population mean estimation of the bus imbalanced power, the sample median is used as the estimation of the population mean.

$$
\hat{\mu}_{j}=\left\{\begin{array}{c}
Y_{j}^{\left(\frac{n+1}{2}\right)} \quad n=2 \mathrm{k}+1 \\
\frac{1}{2}\left(Y_{j}^{\left(\frac{n}{2}\right)}+Y_{j}^{\left(\frac{n}{2}+1\right)}\right) \quad n=2 k
\end{array}\right.
$$

In this formula, $Y_{j}^{(1)}, Y_{j}^{(2)}, \ldots, Y_{j}^{(n)}$ is sample sequence from small to large.

\section{EXAMPLES ANALYSIS}

In order to verify the validity of the method mentioned above, we have made a statistic about the bus imbalanced power in two $500 \mathrm{kV}$ plant stations in North China. The statistics include both the imbalanced active power and reactive power of the 4 bus bar $(500 \mathrm{kV}$ and $220 \mathrm{kV})$ in those two plant stations. The sampling period is 1 minute, and the sampling points are 1440 points. The confidence level is 1$\alpha=0.95$.Statistical results are shown in Table I.

It can be seen from the table I, the deviation between sample mean value and sample median is the largest in $220 \mathrm{kV}$ bus imbalanced reactive power of station B. And the sample standard deviation is outside the confidence interval of the 
standard deviation.

TABLE I. STATISTICAL RESULTS OF BUS IMBALANCED POWER

\begin{tabular}{|c|c|c|c|c|c|}
\hline & Sample mean & Sample median & $\begin{array}{l}\text { Sample standard } \\
\text { deviation }\end{array}$ & $\begin{array}{l}\text { Confidence lower } \\
\text { limit }\end{array}$ & $\begin{array}{l}\text { Confidence upper } \\
\text { limit }\end{array}$ \\
\hline $\begin{array}{c}\text { 220kv bus imbalanced reactive power } \\
\text { of station } A\end{array}$ & -1.23 & -1.22 & 1.79 & 1.72 & 1.86 \\
\hline $\begin{array}{c}220 \mathrm{kv} \text { bus imbalanced active power } \\
\text { of station A }\end{array}$ & -0.4 & -0.36 & 2.24 & 2.16 & 2.33 \\
\hline $\begin{array}{c}500 \mathrm{kv} \text { bus imbalanced reactive power } \\
\text { of station } A\end{array}$ & -1.54 & -1.52 & 1.04 & 1.00 & 1.08 \\
\hline $\begin{array}{c}500 \mathrm{kv} \text { bus imbalanced active power } \\
\text { of station } A\end{array}$ & 0.13 & 0.2 & 1.78 & 1.71 & 1.85 \\
\hline $\begin{array}{c}220 \mathrm{kv} \text { bus imbalanced reactive power } \\
\text { of station B }\end{array}$ & 11.6 & 0.36 & 18.55 & 20.88 & 22.55 \\
\hline $\begin{array}{c}220 \mathrm{kv} \text { bus imbalanced reactive power } \\
\text { of station B (modified) }\end{array}$ & -1.22 & -1.3 & 2.89 & 2.76 & 3.04 \\
\hline $\begin{array}{c}\text { 220kv bus imbalanced active power } \\
\text { of station B }\end{array}$ & 3.26 & 3.3 & 3.37 & 3.24 & 3.50 \\
\hline $\begin{array}{c}500 \mathrm{kv} \text { bus imbalanced reactive power } \\
\text { of station B }\end{array}$ & 7.9 & 8.19 & 5.83 & 5.61 & 6.06 \\
\hline $\begin{array}{c}500 \mathrm{kv} \text { bus imbalanced active power } \\
\text { of station B }\end{array}$ & 7.23 & 7.14 & 14.87 & 14.34 & 15.46 \\
\hline
\end{tabular}

As can be seen from Figure I, the distribution of bus reactive imbalanced power shows multiple wave crests, which does not conform to the normal distribution. After analysis, this result is due to the failure to cancel the measurement atresia while the line maintenance is completed, and the reactive power measurement is always 0 .

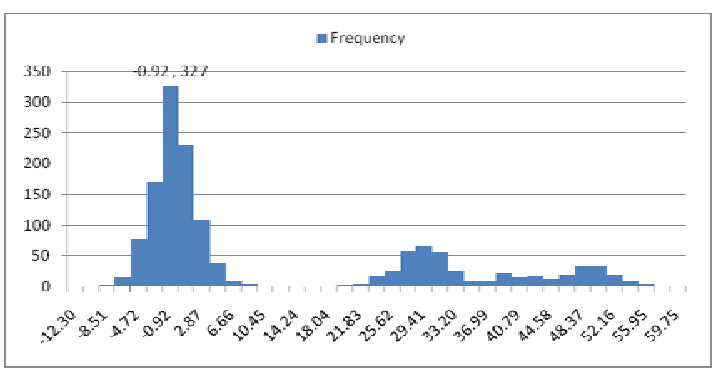

FIGURE I. DISTRIBUTION OF 220KV BUS REACTIVE IMBALANCED POWER IN STATION B

After solving the measurement problem, the distribution of bus imbalanced reactive power in the $220 \mathrm{kV}$ side of the plant station B is shown in Figure II. And this distribution tends to be normal.

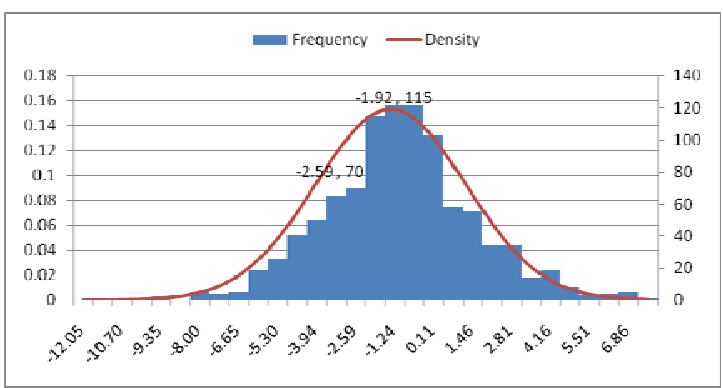

FIGURE II. DISTRIBUTION OF 220KV BUS IMBALANCED REACTIVE POWER IN STATION B (REVISED)
Due to the measurement errors, the sample mean of bus imbalanced reactive power is $11.6 \mathrm{Mvar}$, and the sample median is $0.36 \mathrm{Mvar}$. After solving the measurement problem, the sample mean value is $-1.22 \mathrm{Mvar}$, the sample median is 1.3Mvar, the sample standard deviation and confidence interval tend to be normal. It is thus clear that the sample median is less affected by the measurement error, the larger the sample mean value deviation from the sample median, the greater the probability of abnormal data occurs.

Compared with the $500 \mathrm{kV}$ bus imbalanced active power of station A and station B, the sample mean and sample standard deviation of station Aare smaller than those of station B. It shows that the bus imbalanced power of station $\mathrm{A}$ is more close to 0 , and the distribution is more concentrated.

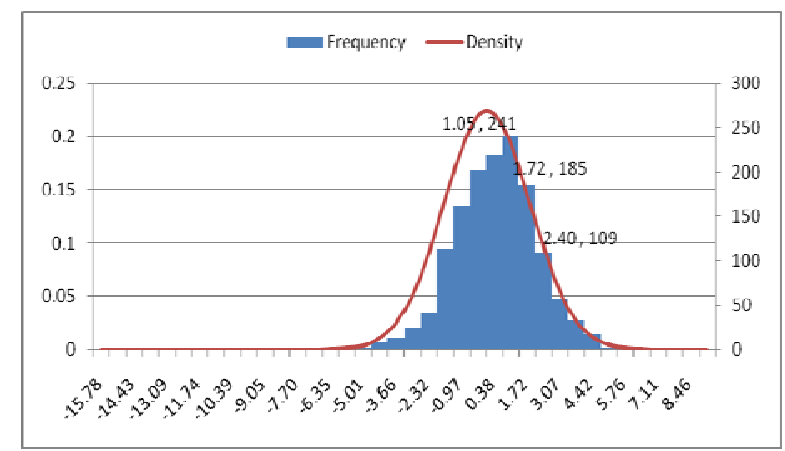

FIGURE III. DISTRIBUTION OF 500KV BUS IMBALANCED ACTIVE POWER IN STATION A 


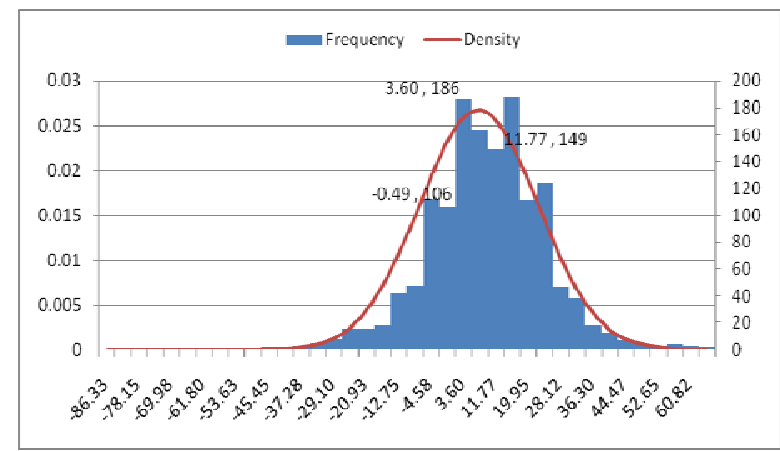

FIGURE IV. DISTRIBUTION OF 500KV BUS IMBALANCED ACTIVE POWER IN STATION B

The distribution of bus imbalanced active power in station $\mathrm{A}$ and $\mathrm{B}$ are respectively shown in Figure III and Figure IV. Therefore, from the view of the imbalanced active power evaluation, the measured quality of plant station $\mathrm{A}$ is better than the plant station B. The problem of plant station B is that the telemetry dead zone is too large, and the telemetry data transmission is delayed.

\section{CONCLUSION}

Due to the random fluctuation of measurement, the evaluation of bus imbalanced power is defective under the single time section. In this paper, the bus imbalanced power is considered as a normal distributed random variable. Using the sample median and sample standard deviation to analyze the quality of plant station telemetry. According to theoretical analysis and practical examples, the mathematical statistic analysis of the bus imbalanced power under the multi time section is proved to have the advantages in reflecting the problem of basic data. Besides, there are many factors that could affect the sample of bus imbalanced power, such as sampling frequency, sampling period, measurement accuracy and transmission network status. So the next subject is how to get accurate and effective samples to better reflect the distribution of bus imbalanced power.

\section{REFERENCES}

[1] ZHAO Weijun, WANG Rongmao, SHI Wenjiang. Methods of improving SCADA analog data's time-synchronization [J]. Automation of Electric Power Systems, 2007, 31(20): 103-107.

[2] GU Quan, CHEN Genjun, CHEN Songlin. A novel state estimation method considering measurement time-delay compensation[J]. Automation of Electric Power Systems, 2009, 33(8): 44-47.

[3] HUANG Yanquan, XIAO Jian, LI Yunfei. A new method to detect and identify bad data based on correlativity of measured data in power System[J]. Power System Technology, 2006, 30(2): 70-71.

[4] LU Zhigang, WANG Haorui, SUN Jikai. Optimal data screening and bad data identification based on sensitive analysis[J]. Power System Technology, 2011, 35(2): 38-42.

[5] WANG Xingzhi, YAN Zheng, SHEN Chen. Power grid bad-data detection and identification based on online kernel learning method [J]. Power System Protection and Control, 2012, 40(1): 50-55.

[6] HE Guangyu, DONG Shufeng. Power system static state estimation based on uncertainty of measurement Part One Result Evaluation[J]. Automation of Electric Power Systems, 2009, 33(9): 21-22.
[7] BAO Fujun, WANG Miao, LI Qiang. Evaluation of power grid data quality based on state estimation results. IFEESM2015, 2015, 385-391.

[8] LI Guangzhen, LIU Wenying, YUN Huizhou. A new data preprocessing method for bus load forecasting[J]. Power System Technology, 2010, 34(2): 149-154. 